\title{
BMJ Open Occupational justice and social inclusion in mental illness and HIV: a scoping review protocol
}

\author{
Clement Nhunzvi, ${ }^{\oplus 1,2}$ Lisa Langhaug, ${ }^{3}$ Edwin Mavindidze, ${ }^{4}$ Richard Harding, ${ }^{\odot 5}$ \\ Roshan Galvaan ${ }^{2}$
}

To cite: Nhunzvi C, Langhaug L, Mavindidze E, et al. Occupational justice and social inclusion in mental illness and HIV: a scoping review protocol. BMJ Open 2019;9:e024049. doi:10.1136/ bmjopen-2018-024049

- Prepublication history and additional material for this paper are available online. To view these files, please visit the journal online (http://dx.doi. org/10.1136/bmjopen-2018024049).

Received 15 May 2018

Revised 13 November 2018

Accepted 21 December 2018

Check for updates

(c) Author(s) (or their employer(s)) 2019. Re-use permitted under CC BY-NC. No commercial re-use. See rights and permissions. Published by BMJ.

${ }^{1}$ Rehabilitation, University of Zimbabwe, College of Health Sciences, Harare, Harare, Zimbabwe

${ }^{2}$ Health and Rehabilitation Sciences, University of Cape Town, Cape Town, South Africa ${ }^{3}$ African Mental Health Research Initiative (AMARI), Department of Psychiatry, University of Zimbabwe College of Health Sciences, Harare, Harare, Zimbabwe

${ }^{4}$ Occupational Therapy, Ingutsheni Central Hospital, Bulawayo, Bulawayo, Zimbabwe ${ }^{5}$ Dept of Palliative Care and Policy, Kings College London, London, UK

Correspondence to Mr Clement Nhunzvi; clemynhu@gmail.com

\section{ABSTRACT}

Introduction Mental illness and HIV remain prevalent as chronic and stigmatised conditions and a global public health concern. Disability-adjusted life-years due to comorbid neuropsychiatric conditions and HIV are rising. Occupational justice and social inclusion emphasise the importance of equity and the utility of resources and opportunities for all to engage in diverse, healthy and meaningful activities. However, succinct conceptualisation of social inclusion and occupational justice, including the relationship between these concepts is still limited. This hampers their effective utilisation in research and practice. Here, we present our scoping review protocol to appraise literature to describe and explain the state of conceptualisation of occupational justice and social inclusion in relation to mental illness and HIV. We are aiming to review the definitions, current utilisation and relationships between occupational justice and social inclusion to inform further theorisation and practice application.

Methods and analysis This scoping review protocol follows existing guidelines for scoping reviews in occupational therapy with particular attention on Arksey and O'Malley's (2005) scoping review framework. We iteratively developed a search strategy and carried out our search using the following databases: PubMed, Scopus, Academic Search Premier, Cumulative Index to Nursing and Allied Health Literature, Africa-Wide Information, Humanities International Complete, Web of Science, PsychInfo and SocINDEX. To enhance the comprehensiveness of our search and capture all relevant information, we will also search a variety of grey literature sources. Two reviewers will independently screen eligible studies for inclusion. Bibliographic data, abstract content and aspects of the study design and findings will be extracted and thematically analysed.

Ethics and dissemination As secondary analysis, this scoping review does not require ethics approval. Results will summarise and disseminate existing research related to occupational justice and social inclusion in mental health and HIV/AIDS care, describing the conceptualisation, relationships between concepts and identifying gaps for further research and practical application. We will disseminate the results through peer-reviewed journals and conferences, targeting clinicians, academics, researchers and policy makers.

\section{Strengths and limitations of this study}

- The breadth of chronic and stigmatised conditions, social inclusion and occupational justice and need for a comprehensive and systematic description of research activity and gaps, make the scoping review a particularly useful methodology.

- A plausible strategy to identify research foci and knowledge gaps related to social inclusion and occupational justice in chronic and stigmatised conditions is presented.

- Based on the multidimensional nature of the concepts under review, methods on how to synthesise research data from diverse designs are put in place.

- Focus is on conceptual and theoretical aspects and not on outcomes of interventions and data synthesis will be limited to work published in English originally or with available English translated copies.

- Focus on mental illness, which is a large grouping of several different conditions, can introduce some generalisation biases; however this will aid in identifying the range of diverse influences of different mental illnesses on concepts under study.

\section{INTRODUCTION}

There is a growing public health concern over the complex relationship between mental illness and HIV. ${ }^{2}$ This is especially so in contexts where the prevalence of HIV among people with mental illness is as high as $25 \%$ in sub-Saharan Africa. ${ }^{34}$ Disability-adjusted life years (DALYs) due to comorbid neuropsychiatric conditions and HIV is rising, with $80 \%$ of DALYs in sub-Saharan Africa being a result of the combined effect of mental illness and HIV. ${ }^{5}{ }^{6}$ Chronic conditions such as mental illness and HIV are still stigmatised and discriminated against, ${ }^{3}$ notably so in comorbid cases, thereby making health and social well-being outcomes fundamental beyond biomedical stabilisation. Developing a better understanding of the occupational justice and social inclusion needs of the affected groups will support intervention development. Occupational 
justice is a growing concept in occupational science and therapy ${ }^{7-9}$ that can help shape care for people who are living with highly stigmatised chronic conditions, including mental illness and HIV. ${ }^{10} 11$ The relevance of occupational justice to highly stigmatised chronic conditions can be traced to its emphasis on social determinants of health and social inclusion as outcomes. ${ }^{11}$ Similarly, social inclusion is receiving increased attention in mental health $^{12}{ }^{13}$ and physical conditions such as HIV. There is a growing emphasis on person-centred outcomes for interventions promoting general and mental health, with social inclusion as the ultimate goal when improving mental health. ${ }^{14}{ }^{15}$ In this context, mental illness implies the diseased state or presence of a mental health disorder, while mental health is defined as a dynamic state of internal equilibrium and well-being which enables individuals to recognise and use their abilities in harmony with the universal values of the society in which they live, cope with the normal stresses of life and makes them economically productive. ${ }^{16}$ Mental health practice refers to the field of practice in occupational therapy and this is how these terms are to be used in this paper.

Occupational justice is defined as equity and fairness for individuals, groups and communities regarding resources and opportunities for their engagement in diverse, healthy and meaningful occupations. ${ }^{717}$ For example, a patient living with a chronic and stigmatised condition who lacks occupational justice (or is subject to occupational injustice) may be denied meaningful employment because of his/her condition, rather than because of his/ her qualifications, or may not be given reasonable accommodation in the workplace. Social inclusion is defined as a multidimensional process or state where prevailing conditions enable full and active participation in all aspects of everyday life. ${ }^{12} 18$ These aspects include civic, social, economic and political activities, as well as participation in decision-making processes irrespective of personal characteristic differences. ${ }^{12}{ }^{18}$ For someone living with a chronic and stigmatised condition, social inclusion would mean availing of resources and opportunities targeting all marginalised groups in that community at a macrolevel. Focusing on social inclusion in the management of mental illness and HIV makes it possible to consider a person's life satisfaction, quality of life, social well-being and equality issues at all levels of their care, a drive that can potentially benefit from occupational justice.

However, to aid our understanding of integration and utilisation of occupational justice and social inclusion in practice, we need to better understand the conceptualisation and relationship between occupational justice and social inclusion. These two concepts are potentially key in informing the promotion of a sustainable person and community-centred recovery with reference to chronic and stigmatised conditions. ${ }^{14} 15$ Despite limited exploration of these concepts in relation to each other, they share notions of human rights and justice, with an emphasis on marginalised and disadvantaged population groups. We aim to review and appraise the definitions, current utilisation and relationships between these concepts. This will inform further theorisation and application in practice of occupational justice and social inclusion in comorbid mental illness and HIV.

\section{METHODS}

Due to the novelty of the area and diverse disciplinary location of existing literature, we propose a scoping review design to achieve our aim. ${ }^{19} 20$ A scoping review is a form of knowledge synthesis that addresses an exploratory research question rather than a highly focused question needed for a systematic review. ${ }^{19}{ }^{20}$ A scoping review aims to map key concepts, types of evidence and gaps in research related to a defined area by systematically searching, selecting and synthesising existing knowledge. ${ }^{20-22}$ The breadth of the area and need for a comprehensive and systematic picture of research activity and gaps, makes scoping review an appropriate methodology for this protocol.

This scoping review protocol follows Arksey and $\mathrm{O}^{`} \mathrm{Mal}-$ ley`s scoping review framework, ${ }^{20}$ guidelines for scoping reviews in occupational therapy ${ }^{21}$ and relevant examples of scoping review protocols. ${ }^{23}{ }^{24}$ The five stages we will follow are:

Stage 1: identifying the research question.

Stage 2: identifying relevant studies.

Stage 3: study selection.

Stage 4: charting the data.

Stage 5: collating, summarising and reporting the results.

Each of the stages planned for the scoping review is described below.

\section{Stage 1: identifying the research question}

An iterative process of refining the research question as a team of authors ${ }^{20}$ was followed in order to generate a guiding research question. The process of refining the question was shaped by reflecting on familiar and available literature in the subject area of occupational justice and social inclusion in occupational therapy, mental health practice and HIV. To facilitate a comprehensive review of the literature, a somewhat broad question was needed, inclusive of the study constructs of interest. ${ }^{20}$ Based on the subject area terminology, literature and our understanding of current practice trends in managing chronic, stigmatised and discriminated-against conditions and the concepts of occupational justice and social inclusion, we asked the following question:

What is the extent of conceptualisation regarding the definitions, current utilisation and relationships between occupational justice and social inclusion in the highly stigmatised and chronic conditions of mental illness and HIV?

Subsequently, the following aim and objectives were formulated:

Aim: to review and appraise the definitions, current utilisation and relationships between occupational justice 
and social inclusion among people with mental illness and HIV and identify areas for further knowledge development and utilisation.

\section{OBJECTIVES}

- To identify existing research that utilised concepts of occupational justice and social inclusion in mental health and HIV care.

- To describe the conceptualisation of and research into the concepts of occupational justice and social inclusion with reference to two highly stigmatised and chronic conditions, that is, mental illness and HIV.

- To identify relationships between occupational justice and social inclusion in the mental health and HIV literature.

- To determine potential areas for further development, integration and application of these concepts in mental health and HIV and AIDS care.

\section{Stage 2: identifying relevant studies}

Although the aim of a scoping review can be broad, parameters must still guide the search strategy. ${ }^{20}$ With the help of a health sciences librarian, the first and last authors developed provisional eligibility criteria, exclusion criteria and identified relevant databases to be searched. This iterative process aimed at identifying a specific search strategy and inclusive search terms. We ran trial searches with different combinations to determine whether our strategy yielded key literature.

The following steps highlight these:

\section{Selection criteria}

The literature search will use the following inclusion criteria to guide the identification of articles to be reviewed.

- Peer-reviewed studies and grey literature in English or with available English translations.

- Literature with a primary focus on evaluating, defining, or exploring the concepts of social inclusion or occupational justice for people living with a mental disorder or HIV will be included. We will include studies and reports that use the terms "social inclusion" or "occupational justice" and their derivatives even if their use of these terms does not conform to standard definitions (Occupational justice: the full enjoyment of occupational rights, implying equity and fairness for individuals, groups and communities regarding their engagement in diverse, healthy and meaningful occupations. ${ }^{4}$ ) (Social inclusion: a multidimensional process or state where prevailing conditions enable full and active participation of every member of the society in all aspects of life, including civic, social, economic and political activities, as well as participation in decision-making processes irrespective of personal characteristics differences. ${ }^{12}$ ). If studies or reports do not include these keywords or
Table 1 General search strategy

\begin{tabular}{ll}
\hline Keyword & Alternative words \\
\hline Occupational therapy & Occupational rehabilitation \\
Mental health & $\begin{array}{l}\text { Mental illness OR Mental disorder } \\
\text { OR Psychiatric disability }\end{array}$ \\
Occupational justice & $\begin{array}{l}\text { Occupational injustice OR } \\
\text { Occupational marginalisation } \\
\text { OR Occupational alienation OR } \\
\text { Occupational imbalance OR } \\
\text { Occupational deprivation }\end{array}$ \\
Social inclusion & $\begin{array}{l}\text { Social exclusion OR Social isolation } \\
\text { OR social integration }\end{array}$ \\
HIV & HIV OR HIV/AIDS OR HIV infection \\
& OR AIDS \\
\hline
\end{tabular}

alternative words as described in table 1 , they will be excluded.

- Both theoretical and empirical literature.

- Literature published in the last two decades (January 1997-December 2017). The last two decades have seen occupational justice make its way into the conceptual debate in occupational science and occupational therapy.

- Studies from any country.

- Studies using both qualitative and quantitative study designs.

\section{Databases}

The following databases will be searched: PubMed, Scopus, Academic Search Premier, the Cumulative Index to Nursing and Allied Health Literature, Africa-Wide Information, Humanities International Complete, Web of Science, PsychInfo and SocINDEX. PubMed is the free platform we will use for searching articles indexed on the Medline database. A librarian used the journal indexing system in identifying suitable databases. These databases were selected to capture a comprehensive sample of literature, both grey and peer-reviewed, from biomedical health sciences, allied health sciences, social sciences and other disciplines. To enhance comprehensiveness of our search and capture all relevant information, we will also search a variety of grey literature sources. We will search grey literature databases like Grey literature Report, Web of Science Conference Proceedings and Open Grey.

\section{Search strategy}

The search strategy evolved during the development of the provisional eligibility criteria of the scoping review. We drew on the occupational justice/injustice terminology and social inclusion/exclusion language in occupational therapy, mental health and HIV practice to come up with operational search terms for occupational justice and social inclusion with reference to chronic and stigmatised conditions. The first and last authors (CN and RG), together with a librarian, in a repeated process, developed 
Table 2 Inclusion and exclusion criteria for acceptable articles

\begin{tabular}{ll}
\hline Criteria for inclusion & Criteria for exclusion \\
\hline Minimum criteria required in the & Used animal \\
abstract: & \\
subjects. \\
Explicit mention of mental \\
Explicit mention of either \\
occupational justice and its \\
varieties or social inclusion \\
and its varieties. (varieties are \\
informed by how articles with \\
these terms are indexed in \\
databases). \\
Date range (1997-2017). \\
English language. \\
English translation of abstract \\
and article available.
\end{tabular}

an inclusive list of primary and secondary search terms and the applicable filtering methods. The primary search terms focused on the primary concepts of occupational justice and social inclusion. The secondary search terms included the broader terms of mental health, occupational therapy, mental illness, HIV and rehabilitation. The main filtering method was that of date range, that is within the last 20 years (1997-2017).

We used our preliminary search to review publication titles which further informed refinement of our search strategy. To come up with an appropriate search strategy that would maximise sensitivity and specificity, our consultant librarian played a key role in determining and pretesting possible keywords. All the search strategies were also modified to comply with the different databases we searched. The suggested Boolean phrases, MESH terms and filters are listed below (Table 1). Online supplementary appendix 1 highlights the preliminary search strategy for PubMed which will be adapted accordingly for other databases.

\section{Stage 3: study selection}

This review will follow a three-part article selection process. Article titles will be reviewed first and the first author $(\mathrm{CN})$ will review the titles to identify articles that i) indicate a research focus on mental illness or HIV or both and ii) titles including the key terms of occupational justice or social inclusion or both. However, where there is uncertainty about the title, especially around the research focus, the article will not be eliminated until it is examined more in depth by looking at the abstract in stage 2 .

The second stage of article selection will be conducted by two independent reviewers, the first and third authors (CN \& EM). This stage will involve review of titles and abstracts using the inclusion and exclusion criteria described in table 2. Any differences arising between the two independent reviews will be resolved with the aid of a third reviewer, who will be second author (LL). What is key in our scoping review is to include abstracts which demonstrate the subject area content being reviewed, that is occupational justice and social inclusion in two highly stigmatised and chronic conditions, mental illness and HIV.

In the third stage, the two independent reviewers $(\mathrm{CN}$ and EM) will each screen the full-text articles to determine if they meet the inclusion/exclusion criteria. Any discordant full-text articles will be resolved through discussion with a third reviewer (LL) until full consensus is reached.

We will use Cohen's $\kappa$ statistic to determine inter-rater agreement. Inter-rater agreement will be calculated at both the title and abstract review stage and at the full article review stage.

\section{Stage 4: charting the data}

Key information about the study content will be extracted. Using a framework specifically designed for this review (table 3), the extraction will include standard bibliometric information and details of the study characteristics. Study objectives will guide the development of the categories of the study characteristics. Key information will be extracted from the main body of the article to sufficiently address the research question. We will be flexible in accommodating additional categories that may emerge during the actual review process which can aid in comprehensively answering the question and addressing study objectives. Ongoing consultation with the team remains key to successful completion of this review and maintaining research rigour. ${ }^{20}$

\begin{tabular}{|c|c|c|}
\hline Bibliometric information & Characteristics of the study & Categories of study characteristics \\
\hline $\begin{array}{l}\text { Study title } \\
\text { Authors } \\
\text { Source/journal } \\
\text { Year of publication } \\
\text { Country } \\
\text { Profession of primary author/academic } \\
\text { discipline } \\
\text { Type of study }\end{array}$ & $\begin{array}{l}\text { Research question/aim(s). } \\
\text { Objective(s). } \\
\text { Intervention(s). } \\
\text { Outcome(s). } \\
\text { Key recommendation(s). } \\
\text { Patient population (eg, diagnosis, } \\
\text { age group, co-morbidity, ethnicity, } \\
\text { etc). }\end{array}$ & $\begin{array}{l}\text { Definition(s) of key concepts and } \\
\text { associated terms - occupational justice } \\
\text { and social inclusion. } \\
\text { Conceptualisation(s) of key concepts. } \\
\text { Summary of key message. } \\
\text { Application to practice. } \\
\text { Links between concepts. } \\
\text { Areas of further development, integration } \\
\text { and application. }\end{array}$ \\
\hline
\end{tabular}


Stage 5: collating, summarising and reporting the results

In this review we will present a narrative account of our findings in two ways. First, a numerical analysis of the bibliometric information will be presented. This will describe the extent, nature and distribution of the studies included in the review through tables and charts mapping bibliometric information and illustrating the academic disciplines in which the study is located, geographic distribution of the scholarship and the research designs applied. Second, the extracted characteristics and categories will be organised thematically using deductive thematic analysis according to different definitions and related concepts for occupational justice and social inclusion. Therefore, the concept definitions will be our primary unit of analysis. With the deductive thematic analyses ${ }^{25}$ we aim to summarise and present definitions, similarities, differences and gaps in conceptualisations of occupational justice and social inclusion for people with mental illness and HIV. Developing the framework for presenting the findings will be an iterative process that will emerge as we examine the articles. ${ }^{20} 2126$

\section{Ethics and dissemination}

Since the scoping review methodology consists of reviewing and collecting data from publicly available literature, this scoping review does not require ethics approval. We aim to summarise and disseminate existing research related to occupational justice and social inclusion in mental illness and HIV/AIDS care, describing the conceptualisation, relationships between concepts and identifying gaps for further research and practical application. We will disseminate the results through peer-reviewed journals and conferences, targeting clinicians, academics, researchers, service users and policy makers.

\section{Patient and public involvement:}

Patient and public involvement representatives have not been directly involved in the design of this scoping review protocol. However, the experience of the first author in working with adults afflicted with HIV and mental health disorders in Zimbabwe informed the need to explore issues faced by this population beyond biomedical care. Life beyond symptom management was not being given the attention it deserved and hence, the need to conduct a scoping review in this area. We also plan to share findings of the planned review with service users, practitioners and policy makers in most accessible formats like newsletters, social media and policy briefs.

\section{CONCLUSION}

To our knowledge, this scoping review, which examines the concepts of occupational justice and social inclusion in populations suffering from two highly stigmatised conditions, is the first of its kind. It has the potential to expand the understanding and utilisation of occupational justice and social inclusion as concepts which promote global mental health. In this scoping review, we aim to review and appraise the definitions, current utilisation and relationships between occupational justice and social inclusion for people with mental illness and HIV. This will give us a better theoretical basis from which to inform further research, practice and training.

Contributors All authors have made substantive intellectual contributions to the development of this protocol. CN and RG conceptualised the review approach and provided general guidance to the research team. Then $\mathrm{CN}$ and $\mathrm{RG}$ were involved in developing the review question and the review design. $\mathrm{CN}$ identified the framework from which $\mathrm{CN}, \mathrm{RG}$ and EM with the help of a subject librarian developed and tested search terms. The first author initially developed the data extraction framework which was then further developed by input from all authors. $L L$ and RH gave substantial review and critique to the draft of the protocol.

Funding This work was supported through the DELTAS Africa Initiative [DEL-1501]. The DELTAS Africa Initiative is an independent funding scheme of the African Academy of Sciences (AAS)'s Alliance for Accelerating Excellence in Science in Africa (AESA) and supported by the New Partnership for Africa's Development Planning and Coordinating Agency (NEPAD Agency) with funding from the Wellcome Trust [DEL-15-01] and the UK government. The views expressed in this publication are those of the author(s) and not necessarily those of AAS, NEPAD Agency, Wellcome Trust or the UK government.

Competing interests None declared.

Patient consent for publication Not required.

Provenance and peer review Not commissioned; externally peer reviewed.

Open access This is an open access article distributed in accordance with the Creative Commons Attribution Non Commercial (CC BY-NC 4.0) license, which permits others to distribute, remix, adapt, build upon this work non-commercially, and license their derivative works on different terms, provided the original work is properly cited, appropriate credit is given, any changes made indicated, and the use is non-commercial. See: http://creativecommons.org/licenses/by-nc/4.0/.

\section{REFERENCES}

1. Bauer-Staeb C, Jörgensen L, Lewis G, et al. Prevalence and risk factors for HIV, hepatitis B, and hepatitis $C$ in people with severe mental illness: a total population study of Sweden. Lancet Psychiatry 2017;4:685-93.

2. Hughes E, Bassi S, Gilbody S, et al. Prevalence of HIV, hepatitis B, and hepatitis $\mathrm{C}$ in people with severe mental illness: a systematic review and meta-analysis. Lancet Psychiatry 2016;3:40-8.

3. Breuer E, Myer L, Struthers H, et al. HIV/AIDS and mental health research in sub-Saharan Africa: a systematic review. Afr J AIDS Res 2011;10:101-22.

4. Dewing S, Plüddemann A, Myers BJ, et al. Review of injection drug use in six African countries: Egypt, Kenya, Mauritius, Nigeria, South Africa and Tanzania. Drugs: Educ Prev Policy 2006;13:121-37.

5. Murray CJ, Vos T, Lozano R, et al. Disability-adjusted life years (DALYs) for 291 diseases and injuries in 21 regions, 1990-2010: a systematic analysis for the Global Burden of Disease Study 2010. Lancet 2012;380:2197-223.

6. Whiteford HA, Degenhardt L, Rehm J, et al. Global burden of disease attributable to mental and substance use disorders: findings from the Global Burden of Disease Study 2010. Lancet 2013;382:1575-86.

7. Durocher E, Rappolt S, Gibson BE. Occupational justice: Future directions. J Occup Sci 2013;21:431-42.

8. Hammell KW. Quality of life, participation and occupational rights: A capabilities perspective. Aust Occup Ther J 2015;62:78-85.

9. Hammell KW. Quality of life, participation and occupational rights: A capabilities perspective. Aust Occup Ther J 2015;62:57-63.

10. Fieldhouse J. Community participation and recovery for mental health service users: An action research inquiry. British Journal of Occupational Therapy 2012;75:419-28.

11. Townsend EA. Boundaries and bridges to adult mental health: Critical occupational and capabilities perspectives of justice. J Occup Sci 2012;19:8-24.

12. Cobigo V, Stuart $\mathrm{H}$. Social inclusion and mental health. Curr Opin Psychiatry 2010;23:453-7.

13. Wright N, Stickley T. Concepts of social inclusion, exclusion and mental health: a review of the international literature. J Psychiatr Ment Health Nurs 2013;20:71-81. 
14. Baumgartner JN, Burns JK. Measuring social inclusion-a key outcome in global mental health. Int J Epidemiol 2014;43:354-64.

15. Patel V, Saxena S, Frankish $\mathrm{H}$, et al. Sustainable development and global mental health-a lancet commission. Lancet 2016;387:1143-5.

16. Galderisi S, Heinz A, Kastrup M, et al. Toward a new definition of mental health. World Psychiatry 2015;14:231-3.

17. Nilsson I, Townsend E. Occupational justice-bridging theory and practice. Scand J Occup Ther 2010;17:57-63.

18. Pereira RB, Whiteford GE. Understanding social inclusion as an international discourse: Implications for enabling participation. $\mathrm{Br} \mathrm{J}$ Occup Ther 2013;76:112-5.

19. Grant MJ, Booth A. A typology of reviews: an analysis of 14 review types and associated methodologies. Health Info Libr J 2009;26:91-108.

20. Arksey H, O'Malley L. Scoping studies: towards a methodological framework. Int J Soc Res Methodol 2005;8:19-32.
21. McKinstry C, Brown T, Gustafsson L. Scoping reviews in occupational therapy: the what, why, and how to. Aust Occup Ther J 2014;61:58-66.

22. Levac D, Colquhoun H, O'Brien KK. Scoping studies: advancing the methodology. Implement Sci 2010;5:69.

23. Halas G, Schultz AS, Rothney J, et al. A scoping review protocol to map the research foci trends in tobacco control over the last decade. BMJ Open 2015;5:e006643-e43.

24. lacono T, Stagg K, Pearce N, et al. A scoping review of Australian allied health research in ehealth. BMC Health Serv Res 2016;16:543-43.

25. Braun V, Clarke V. Thematic analysis. APA handbook of research methods in psychology, Vol 2: Research designs: Quantitative, qualitative, neuropsychological, and biological. 2012:57-71.

26. Cacchione PZ. The evolving methodology of scoping reviews. Clin Nurs Res 2016;25:115-9. 